\title{
Efecto del contacto piel con piel sobre la presencia de síntomas depresivos post parto en mujeres de bajo riesgo obstétrico
}

\author{
ANGELINA DOIS C. ${ }^{1}$, CAMILA LUCCHINI R. ${ }^{1}$ LUIS VILLARROEL D. ${ }^{2}$, CLAUDIA URIBE T. ${ }^{1}$ \\ 1. Escuela de Enfermería, Facultad de Medicina, Pontificia Universidad Católica de Chile, Santiago, Chile. \\ 2. Departamento de Salud Pública, Facultad de Medicina, Pontificia Universidad Católica de Chile, Santiago, Chile.
}

\begin{abstract}
The effect of Mother/Infant skin-to-skin contact on postpartum depressive symptoms in women with low obstetric risk

Background: Postpartum depression can interfere with bonding between the mother and the child. The skinto-skin contact is defined as the contact of the newborn, dry and naked, prone on the mother's bare chest and with a warm blanket placed across the infant's back; this contact takes place immediately after delivery, for at least an hour. Objective: To examine the relationship between skin-to-skin contact and the incidence of depressive symptoms in women with low risk pregnancies. Patients and Method: A prospective analytical study was performed in 393 postpartum women with low obstetric risk in order to evaluate the postpartum depressive symptomatology and its association with biodemographic and skin-to-skin contact variables. Data were collected through a questionnaire at 24 to 48 hours postpartum and through telephone follow-up, at 8 weeks postpartum, in order to screen depressive symptoms using the Edinburgh Depression Scale. Results: $29 \%$ of women reported depressive symptoms. The analysis showed that skin-to-skin contact and early initiation of breastfeeding are significantly associated with the absence of postpartum depressive symptomatology. Conclusion: Skin-to-skin contact was the only variable in this study that can explain the absence of depressive symptoms in women with low risk pregnancies. Skin-to-skin contact implementation is suggested as a preventive strategy.
\end{abstract}

(Key words: Depressive symptoms, postpartum, skin-to-skin contact, Edinburgh Depression Scale).

Rev Chil Pediatr 2013; 84 (3): 285-292

\section{RESUMEN}

Antecedentes: La depresión postparto es un problema de alta prevalencia que impacta en la vinculación afectiva de la madre con su hijo(a). El contacto piel con piel se define como el contacto del recién nacido seco y desnudo, en posición prona sobre el pecho desnudo de su madre, cubierto por su espalda con una manta tibia,

Recibido el 12 de julio de 2012, devuelto para corregir el 29 de agosto de 2012, segunda versión 28 de octubre de 2012 , aceptado para publicación el 25 de febrero de 2013.

Financiamiento: Proyecto FONDEF D07i1046. CONICYT, Pontificia Universidad Católica de Chile y Servicio de Salud Metropolitano Sur Oriente.

Este trabajo cumple con los requisitos sobre consentimiento /asentimiento informado, comité de ética, financiamiento, estudios animales y sobre la ausencia de conflictos de intereses según corresponda.

Correspondencia a:

Angelina Dois C.

E-mail: adois@uc.cl 
inmediatamente ocurrido el parto durante al menos una hora. Objetivo: Examinar la relación entre el contacto piel con piel y la incidencia de síntomas depresivos en mujeres con embarazos de bajo riesgo obstétrico. Pacientes y Método: Estudio analítico prospectivo de 393 puérperas de bajo riesgo obstétrico que evaluó sintomatología depresiva puerperal y su asociación con variables bio-sociodemográficas y características del contacto piel con piel como variable en estudio. Los datos se recolectaron a través de un cuestionario a las 24-48 h postparto y un seguimiento telefónico para pesquisar síntomas depresivos a través de la Escala de Depresión de Edimburgo a las 8 sem postparto. Resultados: Se reporta una incidencia de 29\% de síntomas depresivos. El análisis mostró que el contacto piel con piel y el inicio precoz del amamantamiento están asociado significativamente con la ausencia de sintomatología depresiva postparto. Conclusión: El contacto piel con piel fue la única variable en este estudio capaz de explicar la ausencia de síntomas depresivos en mujeres que cursaron embarazos de bajo riesgo obstétrico. Se sugiere su implementación como estrategia preventiva.

(Palabras clave: síntomas depresivos, post parto, contacto piel con piel, Escala de Depresión de Edimburgo). Rev Chil Pediatr 2013; 84 (3): 285-292

\section{Introducción}

La depresión post parto (DPP) es el trastorno del ánimo más común del puerperio'1. A nivel mundial se calcula que afecta alrededor del $19,2 \%$ de las mujeres en el primer trimestre postparto ${ }^{2}$. En Chile las cifras en población general varían entre un $27,7 \%$ y $41,3 \%{ }^{3}$. Su diagnóstico confiere dificultades, confundiéndose sus síntomas con la adaptación normal al puerperio, lo que provoca bajas tasas de diagnóstico y tratamiento ${ }^{3}$. Entre los factores asociados a estos cuadros están entre otros, los propios de la adaptación materna a la etapa, como la percepción de autoeficacia, la regulación de ritmos fisiológicos del recién nacido (RN) y la instalación de una lactancia materna exitosa ${ }^{1,4,5}$. El contacto piel con piel entre el niño(a) y su madre, es decir, el contacto del $\mathrm{RN}$ seco y desnudo, en posición prona sobre el pecho desnudo de su madre, cubierto por su espalda con una manta tibia, inmediatamente ocurrido el parto durante al menos una hora, favorece la regulación de procesos adaptativos extrauterinos, la instalación de una lactancia materna exitosa, la vinculación afectiva, la disminución de la ansiedad materna y su mayor percepción de autoeficacia ${ }^{6,7}$. Contar con estudios de este tipo es relevante dado que la depresión es la primera causa de años de vida saludables perdidos en las mujeres y la primera causa de discapacidad severa en actividades de la vida diaria en el postparto, con una tasa de subdiagnóstico cercana al $50 \%{ }^{3}$. Por otro lado, el contacto piel con piel entre la madre y su RN es una intervención segura y de bajo costo que ha demostrado tener beneficios para ambos no sólo en el postparto inmediato sino que también en indiciadores de desarrollo infantil saludable ${ }^{6}$.

El objetivo de este trabajo es examinar la relación entre el contacto piel con piel y la incidencia de síntomas depresivos en embarazadas de bajo riesgo obstétrico.

Los datos reportados son parte de la información obtenida durante el desarrollo de un ensayo clínico aleatorio para la asistencia integral del parto en el sistema público de salud (Proyecto FONDEF D07i1046).

\section{Pacientes y Método}

\section{Diseño}

Estudio analítico prospectivo que evaluó sintomatología depresiva puerperal y su asociación con variables bio-sociodemográficas y de las características del contacto piel con piel. Se consideró la variable "contacto piel con piel" como el contacto del RN seco y desnudo, en posición prona sobre el pecho desnudo de su madre, cubierto por su espalda con una manta tibia, inmediatamente ocurrido el parto y durante al menos una hora ${ }^{6}$. Respecto del contacto piel con piel, se evaluó el cumplimiento de 5 dimensiones: a) la inmediatez del contacto entre la madre y su hijo(a) una vez ocurrido el parto y de lo contrario; b) si éste se realizó con el recién nacido desnudo; c) si el pecho materno estaba también desnudo; d) el 
tiempo que duró el contacto, y e) si logró un primer amamantamiento (tabla 1). La hipótesis que guió este estudio fue que el contacto piel con piel entre la madre y su $\mathrm{RN}$, se asocia a la disminución de síntomas depresivos postparto.

\section{Selección y tamaño de la muestra}

El universo correspondió a 998 embarazadas de bajo riesgo obstétrico provenientes de seis centros de salud del área suroriente que tuvieron su parto en una maternidad de Santiago entre agosto de 2010-marzo de 2012. De ellas, se intencionó una muestra de 393 puérperas que comprendían a la totalidad de las mujeres que cumplieron con los requisitos de inclusión: mayores de 15 años, sin diagnóstico previo ni síntomas sugerentes de trastorno del ánimo u otra enfermedad psiquiátrica, que cursaron embarazos de bajo riesgo, dando a luz a $\mathrm{RN}$ únicos, sanos y de término.

\section{Recolección de datos}

Entre 24-48 h postparto un entrevistador capacitado completó un cuestionario especialmente diseñado, que consignaba antecedentes biosociodemográficos, y las características reportadas por la madre sobre su experiencia de contacto piel con piel durante el postparto inmediato (tabla 1). Alrededor de las 8 sem postparto, se hizo un seguimiento telefónico para pesquisar síntomas depresivos (SD) a través de la Escala de Depresión de Edimburgo (EDE) (tabla 1), período que coincide con la máxima prevalencia de DPP y con la disminución de los síntomas propios de un período adaptativo normal ${ }^{8}$. La EDE es un instrumento específico para evaluar severidad sintomática en torno a dos factores principales: tristeza y ansiedad. Su puntaje varía entre 0 y 30 puntos, con una mayor sensibilidad en un punto de corte entre 9 y 10 puntos $^{8-10}$. Las puérperas con puntajes

Tabla 1. Contacto piel con piel. Cuestionario de evaluación

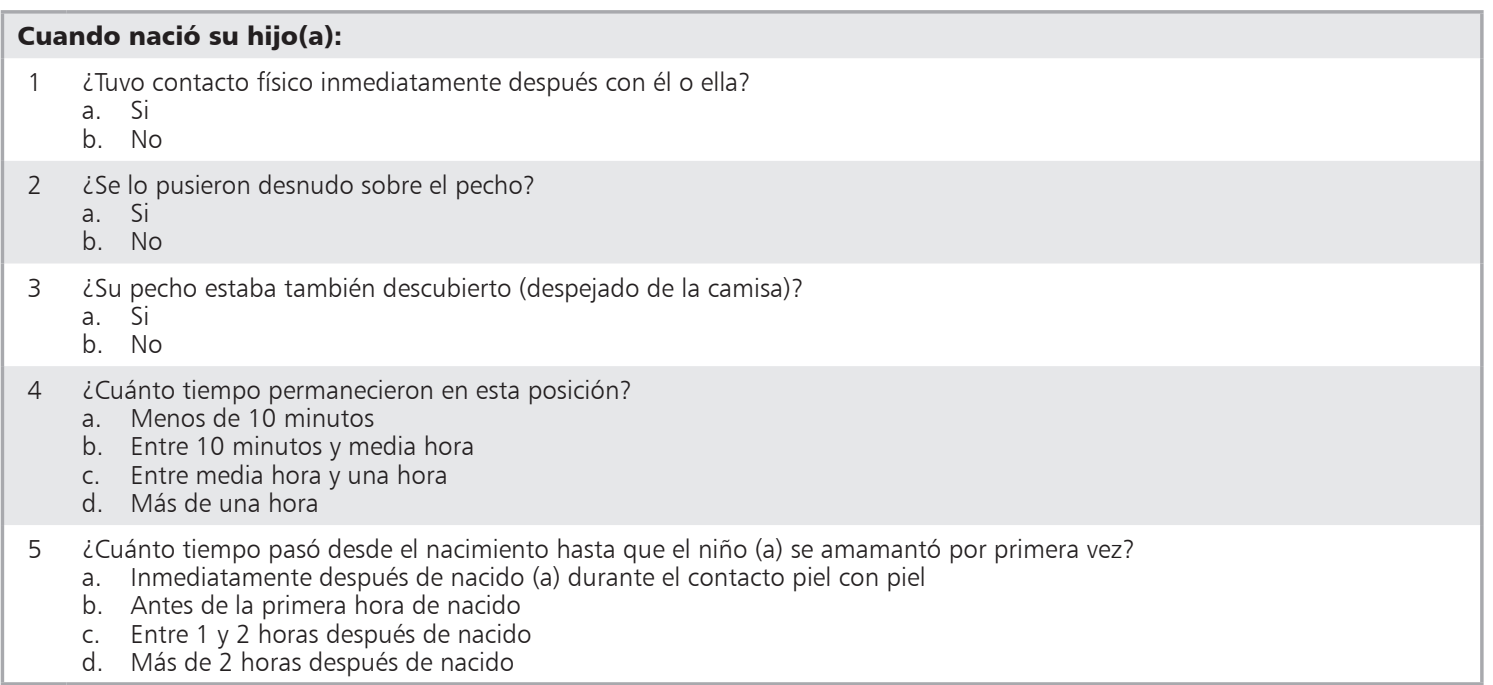

Para que se califique el "contacto piel con piel" como logrado las respuestas deben cumplir los siguientes criterios

\begin{tabular}{|cc|}
\hline Pregunta & Respuesta \\
\hline 1 & a \\
2 & a \\
3 & a \\
4 & d \\
5 & a, bó c \\
\hline
\end{tabular}

Contacto piel con piel

Si:

No: 
Tabla 1. Contacto piel con piel. Cuestionario de evaluación (continuación)

\section{Escala de Depresión de Edimburgo (depresión post natal)}

Las siguientes preguntas hacen referencia a como se ha sentido usted durante los últimos siete días, es decir si hoy es (indicar día: por ejemplo martes), como se ha sentido desde el (indicar día: por ejemplo martes) pasado.

\begin{tabular}{|c|c|c|}
\hline & Pregunta & Puntaje \\
\hline 1 & $\begin{array}{l}\text { He sido capaz de reírme y ver el lado divertido de las cosas: } \\
\text { Tanto como siempre } \\
\text { No tanto ahora } \\
\text { Mucho menos ahora } \\
\text { No, nada }\end{array}$ & $\begin{array}{l}0 \\
1 \\
2 \\
3\end{array}$ \\
\hline 2 & $\begin{array}{l}\text { He disfrutado de mirar hacia adelante: } \\
\text { Tanto como siempre } \\
\text { No tanto ahora } \\
\text { Mucho menos ahora } \\
\text { No, nada }\end{array}$ & $\begin{array}{l}0 \\
1 \\
2 \\
3\end{array}$ \\
\hline 3 & $\begin{array}{l}\text { Cuando las cosas han salido mal, me he culpado a mi misma innecesariamente } \\
\mathrm{Si} \text {, la mayor parte del tiempo } \\
\mathrm{Si} \text {, a veces } \\
\text { No con mucha frecuencia } \\
\text { No, nunca }\end{array}$ & $\begin{array}{l}3 \\
2 \\
1 \\
0\end{array}$ \\
\hline 4 & $\begin{array}{l}\text { He estado nerviosa o inquieta sin tener motivo aparente } \\
\text { No, nunca } \\
\text { Casi nunca } \\
\text { Si, a veces } \\
\text { Si, con mucha frecuencia }\end{array}$ & $\begin{array}{l}0 \\
1 \\
2 \\
3\end{array}$ \\
\hline 5 & $\begin{array}{l}\text { He sentido miedo o he estado asustadiza sin tener motivo: } \\
\mathrm{Si} \text {, bastante } \\
\mathrm{Si} \text {, a veces } \\
\text { No, no mucho } \\
\text { No, nunca }\end{array}$ & $\begin{array}{l}3 \\
2 \\
1 \\
0\end{array}$ \\
\hline 6 & $\begin{array}{l}\text { Las cosas me han estado abrumando } \\
\mathrm{Si} \text {, la mayor parte del tiempo no he podido hacer las cosas en absoluto } \\
\mathrm{Si} \text {, a veces no he podido hacer las cosas tan bien como siempre } \\
\text { No, la mayor parte del tiempo he hecho las cosas bastante bien } \\
\text { No, he estado haciendo las cosas tan bien como siempre }\end{array}$ & $\begin{array}{l}3 \\
2 \\
1 \\
0\end{array}$ \\
\hline 7 & $\begin{array}{l}\text { Me he sentido tan desdichada que he tenido dificultades para dormir: } \\
\text { Si, la mayor parte del tiempo } \\
\text { Si, a veces } \\
\text { No con mucha frecuencia } \\
\text { No, nunca }\end{array}$ & $\begin{array}{l}3 \\
2 \\
1 \\
0\end{array}$ \\
\hline 8 & $\begin{array}{l}\text { Me he sentido triste o desgraciada: } \\
\mathrm{Si} \text {, la mayor parte del tiempo } \\
\mathrm{Si} \text {, bastante a menudo } \\
\text { No con mucha frecuencia } \\
\text { No, nunca }\end{array}$ & $\begin{array}{l}3 \\
2 \\
1 \\
0\end{array}$ \\
\hline 9 & $\begin{array}{l}\text { Me he sentido tan desdichada que he estado llorando: } \\
\text { Si, la mayor parte del tiempo } \\
\text { Si, bastante a menudo } \\
\text { Sólo ocasionalmente } \\
\text { No, nunca }\end{array}$ & $\begin{array}{l}3 \\
2 \\
1 \\
0\end{array}$ \\
\hline 10 & $\begin{array}{l}\text { Se me ha ocurrido la idea de hacerme daño } \\
\text { Si, bastante a menudo } \\
\text { A veces } \\
\text { Casi nunca } \\
\text { Nunca }\end{array}$ & $\begin{array}{l}3 \\
2 \\
1 \\
0\end{array}$ \\
\hline
\end{tabular}

Según los resultados obtenidos se puede clasificar el cuadro en:

\begin{tabular}{|c|c|c|}
\hline & Puntaje & Conducta \\
\hline Sin riesgo de depresión post parto & $\leq 9$ puntos & Control habitual en consultorio \\
\hline En riesgo de depresión post parto & 10-12 puntos & Debe solicitar hora con matrona y avisar que tiene Edimburgo sospechoso \\
\hline Probable depresión & $\geq 13$ puntos & $\begin{array}{l}\text { Debe solicitar hora con médico o psicólogo y avisar que tiene Edimburgo } \\
\text { sospechoso }\end{array}$ \\
\hline
\end{tabular}


sugerentes de DPP fueron derivadas a los Centros de Salud donde se controlaban para su evaluación en profundidad.

\section{Consideraciones éticas}

El Proyecto de Investigación y el Consentimiento Informado fueron aprobados por el Comité de Ética del Servicio de Salud Metropolitano Sur Oriente. Todas las participantes firmaron Consentimiento Informado.

\section{Análisis de datos}

Los resultados de las variables numéricas se presentan como medidas de tendencia central y dispersión y los de las variables categóricas como número de casos y porcentaje. Para la comparación de promedios y de porcentajes se utilizó test t de Student para muestras independientes y test $\chi^{2}$. Para el análisis de asociaciones lineales entre variables numéricas se utilizó correlación muestral de Pearson o por rangos de Spearman, según el origen ordinal o no de las variables involucradas. Por último, con modelos de regresión lineal se determinó la asociación conjunta de dos o más variables con el puntaje de la EDE. Se consideró significativo todo valor $\mathrm{p} \leq 0,05$. Los análisis se hicieron usando el programa estadístico SAS 9.1 para Windows.

\section{Resultados}

Se encuestaron 393 usuarias durante el puerperio inmediato, que al momento de la entrevista no cursaban con sintomatología que permitiera sospechar un cuadro depresivo y que conformaron el grupo de seguimiento a las 8 sem postparto. La edad promedio de las puérperas fue de 24 años. El 53\% tenía escolaridad media completa y en su ma- yoría se desempeñaban como dueñas de casa (55\%). Al momento del parto, el $67,2 \%$ del total vivía con sus parejas. Según antecedentes obstétricos, 162 eran primíparas $(41,2 \%)$ y el $94,9 \%$ del total tuvo un parto vaginal. La edad gestacional promedio fue de $39 \mathrm{sem}$, el peso y talla promedio de los RN fue de 3.426 g y $49,7 \mathrm{~cm}$, respectivamente. El puntaje promedio de APGAR al minuto y 5 min fue de 9 (tabla 2).

La EDE a las 8 sem del postparto, mostró que el 71\% de las encuestadas tuvo menos de 9 puntos, lo que las clasifica como "sin riesgo de depresión". La incidencia de SD fue de $29 \%$, lo que es coincidente con las cifras nacionales al respecto. Dentro de este grupo, $46,5 \%$ tuvo entre 13 y 30 puntos lo que las clasifica dentro de la categoría "probable depresión" y el resto corresponde a la categoría de "en riesgo".

Se analizó la asociación del puntaje de la EDE con algunas variables psicosociales como nivel educacional, tipo de previsión, actividad laboral, hacinamiento, promiscuidad y vivienda entre otras, no encontrándose correlaciones significativas con ninguna de ellas. Situación si-

Tabla 2. Variables perinatales

\begin{tabular}{|c|c|c|c|c|}
\hline Variable & Media & $\begin{array}{l}\text { Desv. } \\
\text { estándar }\end{array}$ & Mínimo & Máximo \\
\hline Edad & 24,3 & 5,7 & 15 & 43 \\
\hline Talla materna, cm & 158,4 & 5,5 & 144 & 174 \\
\hline$N^{\circ}$ hijos vivos & 0,95 & 1,04 & 0 & 5 \\
\hline $\mathrm{N}^{\circ}$ de partos & 0,95 & 1,03 & 0 & 5 \\
\hline Edad gestacional al parto, sem & 39,3 & 1,26 & 37 & 41 \\
\hline Peso RN, $g$ & 3426 & 385,5 & 2.280 & 4.690 \\
\hline Talla RN, cm & 49,7 & 1,7 & 43 & 56 \\
\hline APGAR $1 \mathrm{~min}$ & 8,7 & 0,96 & 2 & 10 \\
\hline APGAR 5 min & 9 & 0,39 & 6 & 10 \\
\hline Peso RN a las 48 hrs, g & 3.235 & 366,8 & 2.330 & 4.520 \\
\hline
\end{tabular}

Tabla 3. Distribución puntaje según Escala de Depresión de Edimburgo (EDE)

\begin{tabular}{|c|c|c|c|c|c|c|}
\hline \multirow{3}{*}{ Puntaje EDE } & \multicolumn{6}{|c|}{ Contacto piel con piel efectivo } \\
\hline & \multicolumn{2}{|c|}{ Sí } & \multicolumn{2}{|c|}{ No } & \multicolumn{2}{|c|}{ Total } \\
\hline & $\mathbf{n}$ & $\%$ & $\mathbf{n}$ & $\%$ & $\mathbf{n}$ & $\%$ \\
\hline $0-9:$ sin riesgo & 121 & 81,2 & 128 & 66,3 & 249 & 72,8 \\
\hline 10-12: en riesgo & 18 & 12,1 & 32 & 16,5 & 50 & 14,6 \\
\hline 13-30: probable depresión & 10 & 6,7 & 33 & 17,1 & 43 & 12,6 \\
\hline Total & 149 & 100 & 193 & 100 & 342 & 100 \\
\hline
\end{tabular}

Frecuencia de valores ausentes: 51 . 
milar ocurrió al analizar la relación entre los puntajes obtenidos y distintas variables biomédicas (edad, tipo de parto, uso de anestesia, paridad, entre otras). Sin embargo, al relacionar los puntajes obtenidos en la EDE con la variable "contacto piel con piel" se observan diferencias estadísticamente significativas entre aquellas madres que tuvieron la posibilidad de establecer contacto piel con piel de aquellas que no lo lograron. E1 33,7\% de las madres que no tuvo contacto piel con piel obtuvo 10 o más puntos en la EDE versus el $18,7 \%$ de las que si lo lograron $(\mathrm{p}<0,01)$ (tabla 3$)$.

$\mathrm{Al}$ analizar las dimensiones del contacto piel con piel, se observa que dos de ellas se relacionan significativamente con los puntajes obtenidos en la EDE (tabla 4). La primera asociada al tiempo en que el $\mathrm{RN}$ estuvo en posición prona en contacto directo con su madre $\left(\chi^{2}=\right.$ $0,018)$. El 67,4\% de las mujeres que reportaron un tiempo $\geq$ a $30 \mathrm{~min}$ tuvieron puntajes menores a 9 puntos en la EDE lo que las sitúa en la categoría de "sin riesgo". De ellas, el $72 \%$ estuvo más de $1 \mathrm{~h}$ en contacto con su hijo(a). En el grupo de mujeres que obtuvo puntajes $\geq 10$ en la misma escala, se observa que sólo el $19,9 \%$ de las que reportaron haber estado en contacto con su hijo(a) por más de $1 \mathrm{~h}$ tienen puntajes sugerentes de un cuadro depresivo, diferencia que fue estadísticamente significativa $(p<0,05)$. La segunda dimensión se relacionó con el tiempo transcurrido entre el nacimiento y el primer amamantamiento del RN $\left(\chi^{2}=0,032\right)$. Del total de madres que alimentaron a sus bebes antes de $1 \mathrm{~h}$, el 76,5\% tuvo puntajes en la EDE $\leq 9$, es decir sin riesgo de DPP, versus el 23,5\% en el grupo que obtiene puntajes $\geq 10$ $(\mathrm{p}<0,05)$. Así mismo, al analizar
Tabla 4. Distribución puntaje Escala de Depresión de Edimburgo (EDE) según sub dimensiones variable "contacto piel con piel"

\begin{tabular}{|lccccccc|}
\hline $\begin{array}{l}\text { Dimensión: "inmediatez } \\
\text { del contacto entre la }\end{array}$ & \multicolumn{3}{c|}{ Puntaje Escala Depresión Edimburgo } \\
$\begin{array}{l}\text { madre y su hijo(a) una } \\
\text { vez ocurrido el parto" }\end{array}$ & $\mathbf{9}$ & $\mathbf{9}$ puntos & $\mathbf{2}$ & $\mathbf{1 0}$ & puntos & \multicolumn{2}{c|}{ Total } \\
Si & 250 & 89,6 & 94 & 82,5 & 344 & 87,5 \\
No & 29 & 10,4 & 20 & 17,5 & 49 & 12,5 \\
Total & 279 & 100 & 114 & 100 & 393 & 100 \\
Frecuencia de valores ausentes: 0 & & & & & & \\
\hline
\end{tabular}

\begin{tabular}{|lccccccc|}
\hline Dimensión: & \multicolumn{4}{c}{ Puntaje Escala Depresión Edimburgo } \\
"se lo pusieron & $\mathbf{5}$ puntos & $\mathbf{2}$ & $\mathbf{1 0}$ & puntos & \multicolumn{2}{c|}{ Total } \\
desnudo sobre su & n & $\mathbf{\%}$ & $\mathbf{n}$ & $\mathbf{\%}$ & $\mathbf{n}$ & $\mathbf{\%}$ \\
pecho" & 245 & 97,6 & 93 & 96,9 & 338 & 97,4 \\
Si & 6 & 2,4 & 3 & 3,1 & 9 & 2,6 \\
No & 251 & 100 & 96 & 100 & 347 & 100 \\
Total & 46 & & & & & \\
\hline Frecuencia de valores ausentes: & 46 & & & & & \\
\hline
\end{tabular}

\begin{tabular}{|lccccccc|}
\hline $\begin{array}{l}\text { Dimensión: } \\
\text { "su pecho estaba } \\
\text { también descubierto" }\end{array}$ & \multicolumn{4}{c}{ Puntaje Escala Depresión Edimburgo } \\
& $\mathbf{9}$ & $\mathbf{9}$ & $\mathbf{n}$ & $\mathbf{\%}$ & $\mathbf{n}$ & $\%$ \\
$\mathrm{Si}$ & 230 & 82,4 & 83 & 72,8 & 313 & 91,3 \\
No & 19 & 17,6 & 11 & 27,2 & 30 & 8,7 \\
Total & 279 & 100 & 114 & 100 & 343 & 100 \\
Frecuencia de valores ausentes: & 50 & & & & & \\
\hline
\end{tabular}

\begin{tabular}{|c|c|c|c|c|c|c|}
\hline \multirow{3}{*}{$\begin{array}{l}\text { Dimensión: "tiempo } \\
\text { en que el RN estuvo en } \\
\text { prono sobre el pecho } \\
\text { desnudo de su madre" }\end{array}$} & \multicolumn{6}{|c|}{ Puntaje Escala Depresión Edimburgo } \\
\hline & \multicolumn{2}{|c|}{$\leq 9$ puntos } & \multicolumn{2}{|c|}{$\geq 10$ puntos } & \multicolumn{2}{|c|}{ Total } \\
\hline & $\mathbf{n}$ & $\%$ & $\mathbf{n}$ & $\%$ & $\mathbf{n}$ & $\%$ \\
\hline$<10 \min$ & 33 & 13,2 & 14 & 15,1 & 47 & 13,7 \\
\hline $10-30 \mathrm{~min}$ & 48 & 19,2 & 31 & 33,3 & 79 & 23,1 \\
\hline $31-60 \mathrm{~min}$ & 47 & 18,9 & 18 & 19,4 & 65 & 19 \\
\hline$>60 \min$ & 121 & 48,6 & 30 & 32,3 & 151 & 44,2 \\
\hline Total & 249 & 100 & 93 & 100 & 342 & 100 \\
\hline Frecuencia de valores ause & 51 & & & & & \\
\hline
\end{tabular}

\begin{tabular}{|c|c|c|c|c|c|c|}
\hline \multirow{3}{*}{$\begin{array}{l}\text { Dimensión: } \\
\text { "tiempo desde el } \\
\text { nacimiento hasta que } \\
\text { fue amamantado" }\end{array}$} & \multicolumn{6}{|c|}{ Puntaje Escala Depresión Edimburgo } \\
\hline & \multicolumn{2}{|c|}{$\leq 9$ puntos } & \multicolumn{2}{|c|}{$\geq 10$ puntos } & \multicolumn{2}{|c|}{ Total } \\
\hline & $\mathbf{n}$ & $\%$ & $\mathbf{n}$ & $\%$ & $\mathbf{n}$ & $\%$ \\
\hline Inmediato & 80 & 29,2 & 32 & 28,6 & 112 & 29 \\
\hline $1<1 \mathrm{~h}$ & 60 & 21,9 & 11 & 9,8 & 71 & 18,4 \\
\hline $1-2 \mathrm{~h}$ & 19 & 6,9 & 10 & 8,9 & 29 & 7,5 \\
\hline$>2 \mathrm{~h}$ & 115 & 42 & 59 & 52,7 & 174 & 45,1 \\
\hline Total & 274 & 100 & 112 & 100 & 386 & 100 \\
\hline \multicolumn{7}{|c|}{ Frecuencia de valores ausentes: 7} \\
\hline
\end{tabular}


el grupo cuyo puntaje es sugerente de un cuadro depresivo, se observa una asociación significativa entre el $61,6 \%$ de las mujeres que alimentaron a su hijo(a) después de $1 \mathrm{~h}$ de ocurrido el parto y el puntaje obtenido en la EDE $(\mathrm{p} \leq 0,05)$. Cabe destacar que la asociación entre puntaje EDE y la posibilidad de la madre de establecer contacto físico inmediato con su $\mathrm{RN}$ no fue significativa en este estudio $\left(\chi^{2}=\right.$ $0,051)$, sin embargo este resultado puede estar asociado al tamaño de la muestra estudiada por lo que pudiera ser objeto de otros análisis en poblaciones de mayor tamaño (tabla 4).

Finalmente, al ajustar modelos de regresión múltiple para determinar si alguna otra variable hacía un aporte en forma conjunta a la explicación del puntaje de la EDE, no se encontraron asociaciones significativas.

\section{Discusión}

El nacimiento de un hijo(a) marca un cambio en la vida de la mujer, siendo descrita como una experiencia psicosocial profunda, que pone a prueba sus competencias personales ${ }^{11}$. Durante el embarazo y puerperio ocurren una serie de cambios que generan una mayor vulnerabilidad para la aparición de trastornos psíquicos en la mujer, siendo la DPP la de mayor prevalencia. La depresión afecta profundamente la auto-percepción y la calidad de vida de las personas. Sin embargo, asociada a la maternidad es particularmente importante sobre todo durante el primer año de crianza de los niños(as) debido a que sus consecuencias muchas veces pueden extenderse a toda la vida ${ }^{12}$.

La DPP puede ocasionar el deterioro de la interacción madre-hijo(a), que se traduce en el establecimiento de apegos inseguros y en el deterioro del desarrollo psicomotor del niño(a). Además, se ha asociado a una mayor posibilidad de desarrollar problemas conductuales a largo plazo y a ser víctimas de violencia intrafamiliar ${ }^{1,4,8}$, a que asistan a un menor número de controles de salud, reciban menor número de inmunizaciones y presenten alteraciones en rutinas de cuidados básicos como por ejemplo, de sueño, higiene y alimentación ${ }^{13}$. Asimismo, se ha descrito en las madres menor percepción de autoeficacia para mantener una lactancia materna exitosa manifestada por un aumento de las preocupaciones en torno a la misma, mayores dificultades y menor satisfacción al amamantar y muchas veces el cese precoz de ésta ${ }^{1,14,15}$

La inicidencia de SD encontrada coincide con los resultados de estudios nacionales que dan cuenta de una prevalencia de DPP que fluctúa entre $27,7 \%$ y $41,3 \%^{3}$, sin embargo, debe considerarse que los resultados obtenidos en este estudio corresponden a mujeres de bajo riesgo obstétrico y sin factores de riesgo aparentes para el desarrollo de un cuadro depresivo postparto, situación que cobra relevancia ya que este grupo, por su condición, habitualmente no es objeto de acciones específicas. La sintomatología depresiva del puerperio en general, se ha asociado fuertemente con los antecedentes de cuadros ansiosos y depresivos previos, con la morbilidad obstétrica y perinatal y con variables como el tipo de parto, la paridad, el nivel educacional y el estado civil entre otras $2,4,5$.

Por otra parte, la literatura reporta que el contacto piel con piel es una intervención mínimamente invasiva, segura para la madre y su hijo(a) si se cautelan las condiciones necesarias, que requiere de bajos costos de implementación y que puede tener un alto impacto en el desarrollo físico y psicosocial posterior del niño(a $)^{6}$. Además, sus beneficios pueden significar la disminución de los costos en salud tanto en lo referente al niño(a) como aquellos derivados del tratamiento del cuadro depresivo materno.

Los resultados de este estudio dan cuenta que el contacto piel con piel fue la única variable que tuvo una asociación significativa con la disminución de la incidencia de SD lo que es coincidente con otros estudios ${ }^{7}$. En Chile, el contacto piel con piel como estrategia de intervención se encuentra respaldada por la política de Protección a la Infancia, Chile Crece Contigo $^{16}$, descrita e indicada en el Manual de Atención personalizado en el proceso reproductivo $^{17}$ y considerada en las Orientaciones para la Planificación y Programación en Red $2012^{18}$, por lo que se sugiere que los Servicios 
de Maternidad públicos y privados que aun no la han incorporado en su práctica habitual, procuren capacitar a los equipos de manera de implementarla. Tal como se ha mencionado, esta intervención ha demostrado ser segura, de bajo costo y no requerir recursos humano y materiales extras a los ya considerados en la atención del proceso de parto ${ }^{6}$.

El contacto piel con piel, que considere el contacto entre la madre y el hijo(a) por al menos una hora y el inicio precoz del amamantamiento, puede constituirse como una estrategia de intervención temprana sobre factores de riesgo modificables, lo que contribuye a mejorar la calidad de vida de las madres y sus hijos(as) y la salud de las familias chilenas.

\section{Referencias}

1.- Dennis C, McQueen K: The relationship between infant-feeding outcomes and postpartum depression: A qualitative systematic review. Pediatrics 2009; 123 : 736-51.

2.- Yim I, Glynn L, Dunkel-Schetter C, Hobel C, ChiczDeMet A, Sandman C: Risk of postpartum depressive symptoms with elevated corticotropin-releasing hormone in human pregnancy. Arch Gen Psychiatry 2009; 66: 162-69.

3.- Ministerio de Salud: Guía clínica: Tratamiento de personas con depresión. Santiago de Chile: Ministerio de Salud; 2006.

4.- Rojas G, Fritsh R, Solís J, González M, Guajardo V, Araya $R$ : Calidad de vida de mujeres deprimidas en el posparto. Rev Med Chile 2006; 134: 713-20.

5.- National Health and Medical Research Council: Postnatal depression. A systematic review of published scientific literature to 1999. Canberra Australia: National Health and Medical Research Council; 2000.

6.- Moore ER, Anderson GC, Bergman N: Early skin to skin contact for mothers and their healthy new born infants. Cochrane Database Systematic Reviews 1999; 3. Art. No.: CD003519.DOI:10.1002/14651858. CD003519.pub2.
7.- Rivara G, Rivara P, Cabrejos K, et al: Contacto piel a piel inmediato: efecto sobre el estado de ansiedad y depresión materna posparto y sobre la adaptabilidad neonatal hacia la lactancia materna precoz. Rev Peru Pediatr 2007; 60: 140-9.

8.- Castañón C, Pinto J: Mejorando la pesquisa de depresión posparto a través de un instrumento de tamizaje, la escala de depresión posparto de Edimburgo. Rev Med Chile 2008; 136: 851-8.

9.- Jadresic E, Araya R, Jara C: Validation of the Edinburgh Postnatal Depression Escale (EDE) in Chilean postpartum women. J Psychosom Obstet Gynaecol 1995; 16: 187-91.

10.- Maroto G, García M, Fernández A: Evaluación del estado del ánimo en el puerperio con la Escala de Depresión Postnatal de Edimburgo. Int J Clin Health Psychol 2004; 5: 305-18.

11.- Callister L, Khalaf I, Semenic S, Kartchner R, Vehvilainen-Julkunen $K$ : The pain perception of culturally diverse women. Pain Management Nursing 2003; 4 : 145-54.

12.- National Health and Medical Research Council (2000). Postnatal depression. A systematic review of published scientific literature to 1999. Canberra: Autor.

13.- Field T: Postpartum depression effects on early interactions, parenting, and safety practices: A review. Infant Behavior and Development 2010; 33: 1-6.

14.- Grote V, Vik T, Von Kries R, et al: Maternal postnatal depression and child growth: A european cohort study. BMC Pediatrics 2010; 10 (14). http://www.ncbi.nlm. nih.gov/pmc/articles/PMC2850333/pdf/1471-2431-1014.pdf, última visita 14-05-2011.

15.- Schwartz K, Hannah J, D'Arcy M: Factors associated with weaning in the first three months postpartum. Journal of Family Practice 2002; 51: 439-44.

16.- Ministerio de Salud: Memoria de Chile Crece Contigo. Cuatro años creciendo juntos. Historia y desafíos. Santiago de Chile: Ministerio de Salud; 2011.

17.- Ministerio de Salud: Manual de atención personalizado en el proceso reproductivo. Santiago de Chile: Ministerio de Salud; 2008.

18.- Ministerio de Salud: Orientaciones para la Planificación y Programación en Red Año 2012. Santiago de Chile: Ministerio de Salud; 2012. 